\title{
TENDÊNCIAS TEÓRICO-METODOLÓGICAS NA PESQUISA EM EDUCAÇÃO: O CASO DO MESTRADO EM EDUCAÇÃO DA UNIVERSIDADE REGIONAL DE BLUMENAU (FURB), SANTA CATARINA, BRASIL
}

\section{Daniela Santini Araújo Adolfo Ramos Lamar}

\section{RESUMO}

O principal objetivo da presente pesquisa consiste em abordar essas tendências na produção de Dissertações de Mestrado no Programa de Pós-graduação em Educação (PPGE) da Universidade Regional de Blumenau (FURB), Blumenau, Santa Catarina, Brasil. A pesquisa é de caráter qualiquantitativo e fundamenta-se no estudo de 44 Dissertações de Mestrado em Educação da FURB relacionadas ao Ensino de Ciência, Filosofia e Epistemologia da Educação. Pode-se observar uma importante influência das perspectivas filosóficas de Paulo Freire e de Thomas S. Kuhn nas Dissertações que têm com foco a Filosofia e Epistemologia da Educação. Já nas Dissertações que têm como problemática de pesquisa o Ensino de Ciências, a maior fundamentação está em Ubiratan D’Ambrósio e Fritjof Capra. Em ambos o caso, prevalece a pesquisa qualitativa. A metodologia de pesquisa utilizada aparece no capítulo introdutório. Observa-se que as Dissertações sobre o Ensino de Ciências colocam de forma mais explícita os autores utilizados na definição da Metodologia da Pesquisa. As tendências teórico-metodológicas da produção de Dissertações do Mestrado em Educação da FURB manifestam a crescente preocupação em refletir sobre os fundamentos da Pesquisa em Educação e sua cientificidade.

\section{PALAVRAS-CHAVE}

Pesquisa em educação; Tendências teórico-metodológicas; Filosofia da educação; Epistemologia da educação; Metodologia da pesquisa

\section{THEORETICAL-METHODOLOGICAL TRENDS IN EDUCATION RESEARCH: THE CASE OF THE MASTER IN EDUCATION COURSE OF BLUMENAU REGIONAL UNIVERSITY (FURB), SANTA CATARINA STATE, BRAZIL}

\begin{abstract}
The main objective of this research is to address these trends in the production of Dissertations in the Masters Program for Post-graduate diploma in Education (PPGE) Regional at the University of Blumenau (FURB), Blumenau, Santa Catarina, Brazil. The search is character-quality quantitative and based on the study of 44 Dissertations of Master in Education of FURB related to the Teaching of Science, Philosophy and Epistemologia Education. You can see a significant influence of philosophical perspectives of Paulo Freire and Thomas S. Kuhn in Dissertations have with the focus Epistemologia Philosophy and Education. Already in Dissertations that are problematic to search the Teaching of Science, the biggest reasons is Ubiratan D'Ambrose and Fritjof Capra. In both cases, the prevailing qualitative research. The methodology used appears in the search introductory chapter. It is observed that the Dissertations on the Teaching of Science put in a more explicit the authors used in the definition of the Research Methodology. The theoretical and methodological trends in the production of Dissertations of the Masters in Education of FURB expressed concern at the increasing reflect on the foundations of Research in Education and its cientificidade.
\end{abstract}

\section{KEYWORDS}

Research in education; Theoretical and methodological trends; Philosophy of Education; Epistemologia of education; Research methodology 


\title{
INTRODUÇÃO
}

Na área de Educação existem muitos apelos para que os professores e estudantes reflitam sobre os processos educacionais. Nesse sentido, uma das principais tarefas do melhoramento da pesquisa em Educação está em refletir sobre os fundamentos teóricometodológicos da pesquisa em Educação.

\begin{abstract}
"Se a pesquisa em educação tendeu a se desenvolver com certas convergências históricas, verifica-se também que ela refletiu, nas décadas assinaladas, modelos de investigação que vinham sendo propostos nos Estados Unidos, Inglaterra ou França, tendo impacto aqui com certo retardo, muitas vezes com uma apropriação simplificada quanto a seus fundamentos.”(GATTI, 2001, p.70)
\end{abstract}

Partindo dessa necessidade, a presente pesquisa teve como principal objetivo identificar as concepções teórico-metodológicas na produção de Dissertações de Mestrado em Educação da Universidade Regional de Blumenau (FURB), Blumenau, Santa Catarina, Brasil. Os objetivos específicos da pesquisa consistiram em verificar a(s) idéia(s) de ciência existente(s) nas dissertações; identificar a(s) metodologia(s) e estrutura(s) das dissertações e oferecer subsídios teórico-metodológicos para a pesquisa no Mestrado em Educação e os Cursos de Licenciatura da FURB.

No período 1993-2005, no Programa de Pós-graduação em Educação da referida universidade (PPGEFURB), foram defendidas um total de 255 Dissertações de Mestrado em Educação. Na presente pesquisa, foi elaborada uma amostra de 44 Dissertações considerando que estas tiveram como principais focos o Ensino de Ciências, Metodologia da Pesquisa, Filosofia da Ciência, Epistemologia, Filosofia e Epistemologia da Educação. As Dissertações de Mestrado selecionadas abordaram importantes problemáticas teórico-metodológicas da área de Educação no Brasil e em nível internacional.

A seleção desta amostra deveu-se, também, ao fato de a bolsista de Iniciação Científica integrar o Grupo de Pesquisa "Filosofia e Educação" (Educogitans), cadastrado no CNPq e pertencente ao Mestrado em Educação da FURB, no qual um dos focos de investigação é a abordagem teórico-metodológica das produções acadêmicas na área da Educação. Deve-se colocar, também, que a bolsista participou do projeto coletivo "A Pesquisa em Educação no Estado de Santa Catarina”. 


\section{O CARÁTER SOCIAL DA CIÊNCIA}

Na pesquisa, constatou-se, através dos autores utilizados, que as Dissertações de Mestrado opõem-se à neutralidade da ciência, defendendo sua dimensão social (ver Tabela $\mathrm{N}^{\circ} 1$ ). Assim, essas dissertações não limitam o condicionamento social da ciência aos aspectos econômicos e políticos, mas também levam em conta as condições sócio-culturais. Nesse sentido, deve-se destacar que na metodologia de pesquisa em educação existem muitas críticas ao economicismo e a subestimação do papel da cultura na produção científica e na Educação Para o filósofo alemão Habermas (1987) existe três tipos de ciência: a empíricoanalítica, a "crítica” e a "fenomenológico-histórica”.

Tabela n. 1.

Tendências teórico-metodológicas na produção de Dissertações do Mestrado em Educação da FURB

\begin{tabular}{|c|c|c|c|c|c|c|c|c|c|}
\hline \multicolumn{2}{|c|}{$\begin{array}{l}\text { Problemas } \\
\text { Filosoficos }\end{array}$} & \multicolumn{2}{|c|}{ Autores filosofia } & \multicolumn{2}{|c|}{ Autores Met. Pesquisa } & \multicolumn{2}{|c|}{ Tipo de pesquisa } & \multicolumn{2}{|c|}{ Lugar da referência } \\
\hline \multirow[t]{4}{*}{15} & \multirow[t]{4}{*}{$100 \%$} & Freire & $7(46.6 \%)$ & Demo & $5(33,3 \%)$ & \multirow{4}{*}{$\begin{array}{l}\text { Qualit. } \\
00\end{array}$} & \multirow{4}{*}{$\begin{array}{l}\text { Quanlit } \\
15 \\
(100 \%)\end{array}$} & \multirow[t]{2}{*}{ Introdução } & \multirow{2}{*}{$\begin{array}{l}12 \\
(80 \%)\end{array}$} \\
\hline & & Khun & $4(26.6 \%)$ & Trivinhos & $2(13,3 \%)$ & & & & \\
\hline & & Gadotti & $2(13,3 \%)$ & Vanconcelos & $2(13.3 \%)$ & & & Fora da & \\
\hline & & Outros & 2 (13.3) & Outros & $6(40,2 \%)$ & & & Intro & $(20 \%)$ \\
\hline 15 & $100 \%$ & & $15(100 \%)$ & & $15(100 \%)$ & & $\begin{array}{l}15 \\
(100 \%)\end{array}$ & & $\begin{array}{l}15 \\
(100 \%)\end{array}$ \\
\hline
\end{tabular}

\begin{tabular}{|c|c|c|c|c|c|c|c|c|c|}
\hline \multicolumn{2}{|c|}{$\begin{array}{l}\text { Problemas } \\
\text { ensino de } \\
\text { ciência }\end{array}$} & \multicolumn{2}{|c|}{ Autores E. Ciências } & \multicolumn{2}{|c|}{ Autores Met. Pesquisa } & \multicolumn{2}{|c|}{ Tipo de pesquisa } & \multicolumn{2}{|c|}{ Lugar da referência } \\
\hline \multirow[t]{4}{*}{29} & \multirow[t]{4}{*}{$100 \%$} & DÁmbrósio & $21(72.4 \%)$ & Demo & $17(58,6 \%)$ & \multirow{4}{*}{$\begin{array}{l}\text { Qualit. } \\
00 \\
(0 \%)\end{array}$} & \multirow{4}{*}{$\begin{array}{l}\text { Quanlit } \\
29 \\
(100 \%)\end{array}$} & \multirow[t]{2}{*}{ Introdução } & \multirow{2}{*}{$\begin{array}{l}22 \\
(75 \%)\end{array}$} \\
\hline & & Capra & $8(27.6 \%)$ & Chizzotti & $5(17,2 \%)$ & & & & \\
\hline & & Assmann & $3(10,3 \%)$ & Menga & $3(10.3 \%)$ & & & Fora da & \\
\hline & & Outros & $2(13.3 \%)$ & Outros & $6(40,2 \%)$ & & & Int & (2 \\
\hline 29 & $100 \%$ & & $29(100 \%)$ & & $29(100 \%)$ & & $\begin{array}{l}29 \\
(100 \%)\end{array}$ & & $\begin{array}{l}29 \\
(100 \%)\end{array}$ \\
\hline
\end{tabular}

A concepção da ciência do físico, historiador e filósofo da ciência Thomas S. Kuhn teve uma considerável influência nas dissertações de Mestrado pesquisadas. Na área de Educação, a teoria “kuhniana” da ciência tem uma grande influência. Esta perspectiva tem como principais fontes teóricas a Filosofia da Ciência do médico Lüdwig Fleck com seus conceitos de "estilo de pensamento" e "coletivo de pensamento” e a Epistemologia Histórica do cientista e filósofo francês Gaston Bachelard com suas idéias de "saltos" e "vigilância 
epistemológica”. Assim, para Bachelard temos que ficar atentos para as Filosofias da Ciência e/ou Epistemologias que permeiam as pesquisas científicas.

T. S. Kuhn é um dos mais renomados estudiosos da ciência pela grande difusão de seus conceitos de paradigmas (modelo de solução de problemas), matriz disciplinar (conceito que tenta responder aos críticos do conceito de paradigma), comunidade científica (grupos de cientistas que defendem determinado paradigma), ciência normal (significa que a comunidade científica tem paradigma dominante), anomalias (elementos que não podem ser explicados pelo paradigma usado pela comunidade científica), crise (as anomalias contribuem para que a comunidade científica perca a confiança nos paradigmas que está usando), ciência revolucionária (a nova ciência que aparece com as mudanças de paradigmas) e incomensurabilidade (a impossibilidade de comparar a teoria anterior com a teoria nova).

O conceito "kuhniano" de paradigma foi o mais usado pelas Dissertações de Mestrado em Educação da FURB que formaram parte da produção científica analisada. Mas, este estudioso defende, também, que na hora de analisar as pesquisas científicas e a própria Educação deve ser considerada os fatores culturais e psicológicos. Nesse caminho, cabe destacar que na pesquisa em Educação existe uma grande debate sobre a relação teoria-fato. Alguns pesquisadores educacionais consideram mais os “fatos" e outros pesquisadores educacionais defendem que o referencial teórico é o que determina os processos de pesquisa científica e a própria explicação da realidade educacional. Assim, cabe destacar que outra importante idéia "kuhniana” consiste em ressaltar o papel da teoria nas revoluções científicas e na Educação.

“[...] as mudanças de paradigma realmente levam os cientistas a ver o mundo definido por seus compromissos de pesquisa de uma maneira diferente. Na medida em que seu único acesso a esse mundo dá-se a través do que vêem e fazem, poderemos ser tentando a dizer que, após uma revolução, os cientistas reagem a um mundo diferente [...] Transformações dessa natureza, embora usualmente sejam mais graduais e quase sempre irreversíveis, acompanham comumente o treinamento científico. Ao olhar uma carta topográfica, o estudante vê linhas sobre o papel; o cartográfico vê a representação de um terreno [...] Somente após várias dessas transformações de visão é que o estudante se torna um habitante do mundo do cientista, vendo o que o cientista vê respondendo como o cientista responde. Contudo, este mundo no qual o estudante penetra não está fixado de uma vez por todas, seja pela natureza do meio ambiente, seja pela ciência [...] Consequentemente, em períodos de revolução, quando a tradição científica normal muda, a percepção que o cientista tem de seu meio ambiente deve ser reeducada.” (KUHN, 1995, p. 46). 
Estudiosos da pesquisa em Educação Brasileira como Sánchez Gamboa (1987), Costa (1994) e Paraíso (2004) destacam as mudanças paradigmáticas nas tradições teóricometodológicas e seus impactos nas idéias de ciência na área de Educação. Em nível internacional, o trabalho de Keiner e Schriewer (1997) coloca como as tradições intelectuais influem nas diferentes trajetórias e autoridade científica das áreas de Educação da França e Alemanha. Cabe aqui ressaltar que o estatuto epistemológico da Educação está muito relacionado com as tradições filosóficas dos países e instituições e o PPGE-FURB não está livre dessa influência.

\footnotetext{
"Se a história das ciências da educação remete, de forma irrecusável, para a das ciências humanas, resta apurar dentro de que medida e com que estatuto aquelas permanecem dentro destas [...] Para melhor se compreender o sentido desta dualidade estatutária, forçoso será inseri-lo no próprio percurso históricoepistemológico das jovens ciências da educação.” (CARVALHO, 1996, p.22)
}

\section{A CRÍTICA DA CIÊNCIA COMO CONHECIMENTO SUPERIOR}

A teoria da Educação de Paulo Freire (1975 e 1998) tem uma importante influência na produção científica do Mestrado em Educação da FURB que foi pesquisada (Ver Tabela N.1). As idéias “freiranas” de “círculos de cultura” e “problemas geradores” são muito importantes para realizar pesquisas educacionais. Assim, nas Dissertações de Mestrado em Educação pesquisadas, existe a preocupação em não considerar os conhecimentos científicos como superiores. Nesse sentido, esta perspectiva chama para considerar os conhecimentos e saberes de todas as pessoas.

Na pesquisa observou-se, também, a crescente influência da algumas teorias "póskuhnianas” Uma dessa perspectivas é a da transdisciplinaridade representada por autores como Ubiratan D’Ambrósio - um dos fundadores do Mestrado em Educação da FURB e um dos mais renomados intelectuais brasileiros, e Fritjof Capra. Outra influente perspectiva é a Epistemologia da Complexidade de Edgar Morin (1994). Este estudioso é um grande crítico de epistemólogos famosos como Kuhn, Lakatos, Popper e Feyerabend por ter tratado de forma marginal à problemática da complexidade. 


\begin{abstract}
"Qual é o erro do pensamento formalizador quantificador que dominou as ciências? Não é, de maneira nenhuma, o facto de um pensamento formalizador e quantificador, não é, de maneira nenhuma, o facto de pôr entre parêntese o que não é quantificável nem formalizável. É facto de ter acabado por acreditar que o que não era formalizável não existia onde não estava mais que a espuma do real. Sonho delirante, porque nada é mais louco que a coerência abstracta. É necessário reencontrar o caminho de um pensamento multidimensional que, evidentemente, integre e desenvolva formalização e quantificação, mas que não se fecha dentro delas." (MORIN, 1994, p.147)
\end{abstract}

Na pesquisa verificou-se, também, que a literatura de Metodologia da Pesquisa é predominante brasileira. Isso é de grande importância para evitar o uso mecânico de referenciais teóricos na pesquisa da problemática educacional brasileira. Umas das principais preocupações da produção científica na área de Educação Brasileira, está em aprofundar os conflitos sobre qual deve ser a estrutura dos trabalhos de conclusão de Curso. Nesse contexto, um dos principais debates está em determinar em qual parte dos Trabalhos de Conclusão de Curso (TCC), Dissertações de Mestrado, Teses de Doutorados e outros tipos de monografias deve estar o capítulo ou item de Metodologia de Pesquisa. Assim, na amostra observamos que a maioria das Dissertações colocou a metodologia de pesquisa na Introdução (Ver Tabela 1).

Outra tendência observada na amostra consiste em que todas as dissertações destacaram que tinham um caráter quali-qualitativo. Esta tendência revela que na produção científica analisada predomina o reconhecimento da dialética entre os aspectos quantitativos e qualitativos. Durante muito tempo, na área de Educação predominou a consideração dos aspectos quantitativos e os aspectos qualitativos eram desconsiderados no planejamento, operacionalização, comunicação e avaliação das pesquisas científicas. Esta posição teóricometodológica positivista tinha, entre seus objetivos, demonstrar a cientificidade da área de Educação.

Nas décadas de 70 e 80, as pesquisas qualitativas ganharam um importante peso nas Ciências Humanas e Sociais. Mas, na área de Educação Brasileira foi na década de 90 que esta tendência ganhou maior força. Porém, deve-se destacar que em alguns momentos existiu a tendência de não considerar os aspectos quantitativos. Assim, é louvável que na produção de Dissertação de Mestrado em Educação da FURB predomine a crítica dessas posições extremas e exista o reconhecimento da necessidade de considerar a dialética entre os aspectos qualitativos e quantitativos. 


\section{A GÜISA DE CONCLUSÃO}

Mapear as concepções que permeiam a produção científica é uma tendência nacional e internacional. Os mapeamentos das perspectivas contribuem para a identificação e análise dos “obstáculos epistemológicos” e fornecem subsídios para o fortalecimento do ensino, pesquisa e extensão na universidade e o melhoramento das relações com a sociedade. O próprio caráter regional da FURB exige uma pesquisa que responda às necessidades teórico-práticas do ensino, em seus diferentes níveis, e de Dissertações que não tenham um caráter cientificista e não estejam defasadas “epistemologicamente”.

Em linhas gerais, as tendências teórico-metodológicas na produção de Dissertações de Mestrado no PPGE-FURB refletem as Epistemologias e Filosofias da Educação predominantes na história dos Programas de Pós-graduação em Educação no Brasil: positivista, crítico-dialética e fenomenológica. Porém, na produção analisada existe uma grande crítica da concepção positivista da ciência e um crescente trabalho com autores de relevância como Paulo Freire, Thomas. S. Kuhn, Edgar Morin, Ubiratan D’Ambrósio e Fritjof Capra.

Um dos possíveis desdobramentos da presente pesquisa consistirá em aprofundar a incorporação de novas tendências à produção científica do PPGE, como é o caso das teorias pós-críticas e os Estudos Sociais da Ciência e da Tecnologia (ESCT).

\section{REFERÊNCIAS}

CARVALHO, A. D. de. Epistemologia das ciências da educação. Porto: Edições Afrontamento, 1996.

COSTA, M. C. V. A Pesquisa em educação: concepções de ciência, paradigmas teóricos e produção de conhecimento. Cadernos de Pesquisa, São Paulo, n. 90, 15-20, agosto, 1994.

FREIRE, P. Pedagogia do oprimido. 3. ed. Rio de Janeiro: Paz e Terra. 1975.

. Pedagogia da autonomia: saberes necessários à prática educativa. 7. ed. Rio de Janeiro: Paz e Terra. 1998. 
GATTI, B. Implicações e perspectivas da pesquisa educacional no Brasil contemporâneo. Cadernos de Pesquisa, São Paulo, n.113, jul. 2001.

HABERMAS, J. Conhecimento e interesse. Rio de Janeiro: Guanabara, 1987.

KEINER, E.; SCHRIEWER, J. Pautas de comunicación y tradiciones intelectuales en las ciencias de la educación: Francia y Alemania. Revista Mexicana de Investigación

Educativa, v.2, n.3 , ene.- jun. 1997. Disponível em: <http://www.comie.org.mx/v1/revista/> Acesso em: 4 mai. 2006.

KUHN, T. S. A estrutura das revoluções científicas. São Paulo: Perspectivas S. A ., 1995.

MORIN, E. Ciência com consciência. Europa - América: Publication Mem Martins, 1994.

PARAÍSO, M. A. Pesquisas pós-críticas em educação no Brasil: esboço de um mapa. Revista Cadernos de Pesquisa, v.34, n.122, São Paulo, 283-303, maio/ago. 2004.

POPKEWITZ, T. S. Lutando em defesa da alma do professor: a política de ensino e a construção do professor. Porto Alegre: Artmed, 2001.

SÁNCHEZ GAMBOA, S. Epistemologia da pesquisa em educação: estruturas lógicas e tendências metodológicas. 229 fl. 1987. Tese (Doutorado em Educação) - Faculdade de Educação, Universidade Estadual de Campinas, Campinas, 1987. 


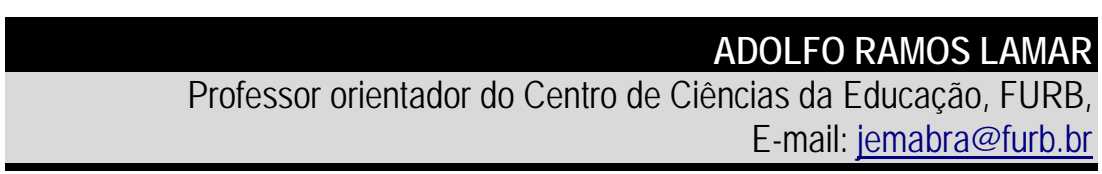

Recebido em: 25/07/2008

Publicado em: 31/01/2009 